Relations industrielles

Industrial Relations

\title{
Leclerc, Michel et Michel Quimper, Les relations du travail au Québec, 2 e édition
}

\section{Jean Boivin}

Volume 55, numéro 2, 2000

URI : https://id.erudit.org/iderudit/051316ar

DOI : https://doi.org/10.7202/051316ar

Aller au sommaire du numéro

Éditeur(s)

Département des relations industrielles de l'Université Laval

ISSN

0034-379X (imprimé)

1703-8138 (numérique)

Découvrir la revue

Citer ce compte rendu

Boivin, J. (2000). Compte rendu de [Leclerc, Michel et Michel Quimper, Les relations du travail au Québec, 2e édition]. Relations industrielles / Industrial Relations, 55(2), 362-364. https://doi.org/10.7202/051316ar

Tous droits réservés @ C Département des relations industrielles de l'Universite Laval, 2000
Ce document est protégé par la loi sur le droit d'auteur. L’utilisation des services d'Érudit (y compris la reproduction) est assujettie à sa politique d'utilisation que vous pouvez consulter en ligne.

https://apropos.erudit.org/fr/usagers/politique-dutilisation/ 


\section{Les relations du travail au Québec, $2^{e}$ édition}

par Michel LECLERC et Michel QUIMPER, Sainte-Foy : Presses de l'Université du Québec, 2000, 403 p., ISBN 2-7605-1059-X.

Ce volume est une réédition de Les relations du travail au Québec: une analyse de la situation dans le secteur public, paru en 1994 chez le même éditeur. Pour cette nouvelle édition, les auteurs ont choisi de retirer le sous-titre «Une analyse de la situation du secteur public $»$. Ceci est un choix fort judicieux car le volume n'y consacre qu'un seul de ses quatorze chapitres. De plus, l'analyse à laquelle la première édition faisait référence, et qui est reproduite intégralement dans la nouvelle édition, n'est en fait que la simple description formelle du cadre légal des relations du travail et des structures de négociation. On ne retrouve même pas une allusion au fait qu'entre 1989 et 1998 le régime de négociation des conventions collectives a été littéralement mis en veilleuse parce que les gouvernements successifs ont préféré s'attaquer à leurs problèmes financiers ou constitutionnels, plutôt que de s'engager dans le fastidieux processus de renouvellement des conventions collectives. Les seuls éléments d'analyse se résument à mentionner que l'une des règles prévues dans le nouveau cadre légal introduit en 1985, la non-négociabilité des salaires pour les deuxième et troisième années des conventions collectives, ne s'est jamais réalisée en pratique et à souligner que la décentralisation des structures de négociation dans les secteurs de la santé et de l'éducation serait souhaitable.

Outre l'ajout de quatre annexes, la structure générale du volume est la même que celle de la première édition. L'ouvrage comporte une introduction et quatre parties. L'introduction porte sur la discipline des relations industrielles et ses paradigmes et écoles de pensée. Les auteurs utilisent ici le modèle systémique de Dunlop pour représenter les phénomènes de relations du travail. Ceci est tout à fait légitime, mais on peut leur reprocher d'avoir raté une excellente occasion de mentionner l'existence de l'approche des choix stratégiques de Kochan, Katz et McKersie. Ceci est d'autant plus surprenant que la contribution de ces auteurs est mentionnée à plusieurs reprises dans le corps du volume.

La première partie traite de l'histoire des relations du travail et comporte quatre chapitres portant sur l'histoire des relations du travail en Grande-Bretagne, en France, aux États-Unis, au Canada et au Québec. On aurait facilement pu faire l'économie des événements qui se sont déroulés dans les trois premiers pays pour analyser plus en profondeur ceux qui sont survenus en contextes canadien et québécois. Les propos demeurent à un niveau trop superficiel pour intéresser véritablement le lecteur. Une annexe comportant des dates et des événements aurait sans doute suffi pour présenter les informations pertinentes.

La deuxième partie est consacrée à la législation du travail. Elle comporte également quatre chapitres traitant du cadre constitutionnel, des lois du travail (normes du travail, santé et sécurité au travail, accidents du travail et maladies professionnelles), du Code du travail et des secteurs public et parapublic. Ces chapitres permettent au lecteur d'obtenir l'essentiel du contenu des diverses législations qui constituent l'épine dorsale des relations du travail. Ils sont écrits dans un langage simple qui les rend accessibles à un vaste public, contrairement à la plupart des manuels de droit du travail qui sont habituellement écrits dans un langage aride et hermétique. On peut cependant déplorer que les auteurs s'en soient tenus à une approche descriptive et qu'ils n' aient pas cru bon d'ajouter des références jurisprudentielles ou des commentaires analytiques, notamment dans le chapitre portant sur le secteur public. 
Dans la troisième partie qui porte sur la vie syndicale au Québec, on retrouve trois chapitres intitulés : « Les organisations syndicales et patronales , « La négociation collective » et « $\mathrm{La}$ gestion de la convention collective $\gg$. Le premier de ces chapitres comporte des statistiques intéressantes sur l'étendue de la représentation syndicale dans différents pays occidentaux ainsi que sur la répartition des effectifs syndicaux au Canada et au Québec selon différents critères (syndicats nationaux et internationaux, régions canadiennes, affiliation selon les centrales syndicales, etc.). La description de la structure et du fonctionnement des organisations syndicales canadiennes est cependant confuse et ne comporte pas suffisamment d'explications pour que le lecteur s'y retrouve. Les auteurs ont bien fait l'effort de reproduire les organigrammes de chacune des centrales québécoises et du Congrès du travail du Canada, mais ils n'y ajoutent qu'une seule page ou un seul paragraphe de texte comme complément d'informations.

Le chapitre suivant sur la négociation collective présente la façon dont les parties se préparent pour négocier, le concept de pouvoir de négociation, la particularité de la négociation collective dans le secteur public par rapport à celle du secteur privé (en termes généraux et non en fonction du contexte québécois) et les défis auxquels cette institution fait face présentement suite à la diminution du pouvoir de négociation des syndicats. Les auteurs soulignent à juste titre que les employeurs et les syndicats sont confrontés à des choix stratégiques difficiles: soit s'engager sur la voie d'une plus grande coopération, soit durcir leurs positions et s'engager sur la voie de la confrontation. Cette dernière option est surtout disponible aux employeurs qui possèdent actuellement un plus grand pouvoir de négociation. Ce chapitre comporte aussi deux nouvelles sections : l'une consacrée aux clauses « orphelins » et l'autre à la négociation raisonnée. Ce dernier sujet est d'ailleurs repris dans une des quatre nouvelles annexes.

Le chapitre sur la gestion de la convention collective est un des meilleurs du volume. Les auteurs analysent systématiquement le contenu de la convention collective et passent en revue ses modalités d'application (procédure de griefs et gestion de la discipline). Cette fois-ci on met la jurisprudence à contribution.

La quatrième partie reprend les trois mêmes chapitres que l'édition précédente, soit « Les nouveaux programmes de gestion des ressources humaines », «L'adaptation aux nouvelles réalités » et « Les prospectives des relations du travail ». Si les trois premières parties du volume étaient consacrées à des sujets étroitement associés aux relations du travail, on peut dire que cette dernière partie présente une allure beaucoup plus hétéroclite. Ainsi, le chapitre 12 aborde des questions telles les programmes de santé et de sécurité au travail, l'accès à l'égalité, la gestion de la carrière, l'aide aux employés, la gestion de la performance, la participation des travailleurs, la qualité de vie au travail, les cercles de qualité, le partenariat, les programmes de communication, la gestion par la qualité totale, le télétravail et la réingénierie. Ces deux derniers sujets n'ont pas été traités dans la première édition. Il faut dire cependant que tous ces sujets ne sont qu'effleurés superficiellement, plusieurs n'étant l'objet que d'un bref paragraphe.

Quant au chapitre 13, il traite de la précarisation de l'emploi, de l'alcool et des autres toxicomanies, des nouveaux types de conflits ainsi que de la société de l'information, un sujet qui n'apparaissait pas dans la première édition.

Le dernier chapitre aborde les perspectives des relations du travail : les nouveaux travailleurs et les nouvelles valeurs de la société, la situation de l'emploi, le contexte organisationnel, la gestion, le syndicalisme, l'état, et les 
relations du travail au début du $X_{X I}{ }^{e}$ siècle. Ces problématiques sont fort pertinentes, mais la façon dont elles sont traitées présente deux inconvénients. D'une part, même si certains passages ont été reformulés pour tenir compte des changements survenus au cours des six années qui se sont écoulées depuis la parution de la première édition, on peut déplorer que la plupart des références soient demeurées les mêmes et que la quasi-totalité datent des années 1980. C'est pourtant un chapitre consacré à la prospection! D'autre part, les nombreuses questions soulevées sur l'avenir du syndicalisme et sur les défis auxquels cette institution est confrontée s'appuient à peu près exclusivement sur des auteurs français tels Crozier, Touraine, Rosanvallon, et Lemattre. Malgré la réputation et le sérieux des auteurs en question, en raison des différences énormes entre les contextes institutionnels des relations du travail en France et au Canada, cette réflexion aurait eu intérêt à être davantage alimentée par des sources nord-américaines, voire québécoises, par exemple le livre de
Murray et Verge (recensé dans $R I / I R$, vol. $55, \mathrm{n}^{\circ} 1$ ).

Les thèmes traités dans les quatre annexes viennent renforcer le caractère hétéroclite de cet ouvrage dont la quatrième partie nous avait déjà donné un certain aperçu. Non pas que des sujets tels l'évolution de la gestion des ressources humaines, l'interrelation entre la GRH, les relations industrielles et la négociation raisonnée, les communications organisationnelles et la résolution des conflits interpersonnels ne soient pas intéressants en soi, au contraire. Mais de les trouver traiter dans un volume qui porte également sur des sujets comme l'histoire des relations du travail dans plusieurs pays, le contenu des principales lois du travail, le fonctionnement des organisations syndicales et patronales ainsi que la négociation et la gestion de la convention collective ne peut que me faire penser à ce proverbe : «Qui trop embrasse, mal étreint ».

\section{JEAN BoIvIN \\ Université Laval}

\section{Legalizing Gender and Equality: Courts, Markets and Unequal Pay for Women in America}

by Robert L. NELSON and William P. BRIDGes, New York: Cambridge University Press, 1999, 393 p., ISBN 0-521-62169-0 (hb).

This is an in depth and critical study of Pay Equity as a law reform movement in the United States since 1970. The book traces the victories and defeats of the past, studies the reasons for the outcomes and then suggests a new methodology of approach.

Analyzing Federal Court decisions dealing with allegations of sex-based pay discrimination, Nelson and Bridges conclude that the Courts' decisions reflect a tension between the law's commitment to equality and the courts' reverence for free markets and efficiency. The authors show how the courts have not countenanced blatant discrimination against women workers. However, the courts have systematically rejected comparable work theories that rely on job evaluation studies. These studies have not been accepted as proof of discrimination and have failed to persuade the courts to oblige employers to correct inequities identified in job evaluation results. Instead, the U.S. courts consistently found that between job pay differentials reflect market wages or "acceptable business judgments" rather than invidious, gender based policies. The authors contend that these judicial decisions reflect the United State's courts' deeply held pro-market ideology but may not be analytically justifiable based on the evidence that had been filed in those cases. 